\title{
Modelling of the kinetics of transesterification reaction of rapeseed oil with different reactant dosing procedures
}

\author{
Jakub Husár ${ }^{1, *}$, Jiři Pecha ${ }^{1}$, Lubomir Š́nek ${ }^{1}$, and Karel Kolomaznik ${ }^{1}$ \\ ${ }^{1}$ Tomas Bata University in Zlín, Faculty of Applied Informatics, Regional Research Centre CEBIA-Tech, Nad Stráněmi 4511,76005 , \\ Zlin, Czech Republic
}

\begin{abstract}
Transesterification of triglycerides is a common method in the production of biodiesel, specifically methyl esters of fatty acids. In this work, the transesterification kinetics was studied for an unusual dosing procedure taking into account the side reaction - hydrolysis. This unwanted side reaction, called saponification, causes the deactivation of the used catalyst and decreases the purity of biodiesel, the main product of transesterification. For these reasons, a model of methanolysis has been designed and clarified considering both the main and side reactions with various dosing of raw material and catalyst. The suggested mathematical model is capable of predicting the state of the reaction mixture in time under different initial reaction mixture composition and different reaction temperature. The verified model could be useful for the estimation of optimal conditions and dosage procedure of methanolysis under which saponification is reduced, and is also important for the design of industrial manufacturing process, equipment and process control algorithms.
\end{abstract}

\section{Introduction}

The instability of the petroleum market, the finite amount of crude oil on the planet Earth and mainly the environmental impact associated with the use of fossil fuels have been a supportive aspect of alternative fuels usage. Biodiesel, containing fatty acid esters, is considered as a viable alternative or additive to petrodiesel due to its environmental benefits, such as renewability, nontoxicity and biodegradability. It is a mixture of fatty acid alkyl esters obtained by transesterification. This chemical process is a reversible reaction where the triglycerides react with short-chain alcohols in the presence of acid or basic catalyst to form fatty acid esters (i.e., biodiesel) and glycerol. Animal fats and vegetable oils could be used as a source for this reaction since these are chemically composed mainly of triglycerides. [1]

This work is based on experimental measurements of the course of the reaction studied, namely methanolysis of rapeseed oil catalyzed by potassium hydroxide. The data served as a basis for the design, evaluation and selection of tested mathematical models. The proposed mathematical models allow the simulation and optimization of the reaction conditions, which is the cornerstone of the design of real industrial reactor control.

\subsection{Biodiesel production}

Biodiesel is usually a product of the process known as transesterification as mentioned above. The scheme of the reaction is shown in Figure 1. Symbols R1, R2 and $\mathrm{R} 3$ represent long hydrocarbon chains, sometimes also called fatty acid chains. The reaction of glycerides (mainly triglycerides) with methanol in the presence of a catalyst gives a mixture of fatty acid methyl esters (FAME) and glycerol (also called glycerin). [2]

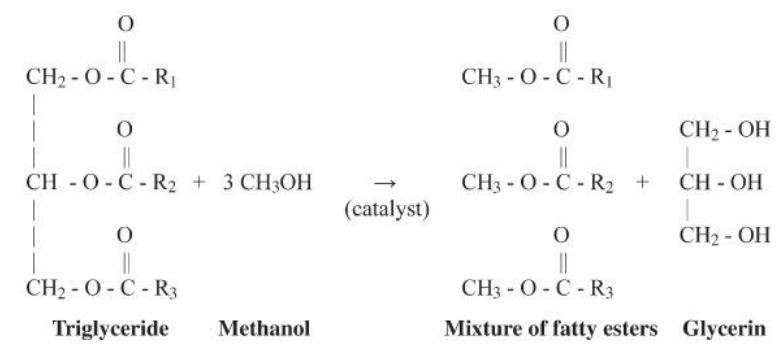

Fig. 1. Chemical reaction - Transesterification. [2]

In a detailed chemical description, it should be noted that triglycerides react sequentially to lower (mono-, di-) glycerides, fatty acid methyl esters and glycerol, as can be seen in Figure 2. [3]

Triglyceride (TG) $+\mathrm{CH}_{3} \mathrm{OH} \leftrightarrow$ Diglyceride $(\mathrm{DG})+\mathrm{RCOOCH}_{3}$ Diglyceride (DG) $+\mathrm{CH}_{3} \mathrm{OH} \leftrightarrow$ Monoglyceride $(\mathrm{MG})+\mathrm{RCOOCH}_{3}$ Monoglyceride $(\mathrm{MG})+\mathrm{CH}_{3} \mathrm{OH} \leftrightarrow$ Glycerol $(\mathrm{G})+\mathrm{RCOOCH}_{3}$

Fig. 2. Detailed description of the reaction. [3]

It is also important to note that the reaction mixture is a two-phase liquid-liquid heterogeneous system, which complicates both the mathematical description and experimental study of this reaction. [4]

\footnotetext{
*Corresponding author: husar@ubt.cz
} 


\subsection{Undesirable effect of a basic catalyst}

The reactions investigated in this paper were catalyzed by potassium hydroxide, a basic catalyst. The undesirable effect of using a basic catalyst is the presence of a side reaction, hydrolysis of glycerides. This side reaction deactivates the catalyst and cleaves the ester bond alongside the transesterification, thus consuming also the fatty acid feed material. Chemically it is a reaction of fatty acid esters with a basic catalyst, resulting in the formation of a molecule of soap together with a molecule of water; thus, the reaction is also called saponification. As can be seen in Figure 3., neutralization of the basic catalyst with free fatty acids is a similar reaction. These reactions are undesirable as the concentration of the catalyst is reduced, resulting in a decrease of the transesterification reaction rate. [2]

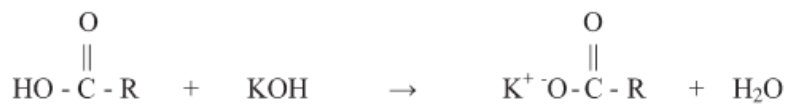

Fatty Acid Potassium Hydroxide Potassium soap Water

Fig. 3. Neutralization of fatty acids. [2]

Although the adverse effects of saponification on the rate of transesterification are known, this side reaction has been only rarely studied in current literature. [3] The purpose of this work was, therefore, to suggest and verify suitable mathematical models for transesterification reaction, which also comprise the side reaction - saponification.

\section{Mathematical description of the reaction}

The following kinetic equation declares the reaction rate dependence on the reaction conditions. The rate of the reaction is a function of concentrations of all reactants and temperature. [5]

$$
r_{A}=-\frac{d c_{A}}{d \tau}=k(T) c_{A}^{\alpha} c_{B}^{\beta}
$$

The reaction rate $r_{A}$ involves concentrations $c$ of the starting substances and the velocity constant $k$, which takes into account the temperature dependence of the reaction rate. Concentration exponents $(\alpha, \beta)$ are determined experimentally. The velocity constant can be calculated according to the Arrhenius equation (2). [5]

$$
k=A \exp \left(-\frac{E^{*}}{R T}\right)
$$

In Arrhenius equation, $E^{*}$ means the activation energy, $A$ is a frequency factor and $R$ is the universal gas constant. The temperature $T$ has a significant influence on the reaction rate. The relationship suggests that reactions with high activation energy are very sensitive to temperature changes, while reactions with lower activation energy are relatively temperature-insensitive. In elemental reactions, the values of the activation energy are positive. The activation energy can be imagined as the energy that must be supplied to the reacting molecules to stimulate the valence electrons. [5]

The Arrhenius equation can be reparametrized by the definition of the reference temperature. This reparametrization simplifies the estimation and interpretation of the parameters and allows to minimize correlation of the parameters. For dynamic models where an analytical solution is not available, a numerical procedure can be used to determine the optimum reference value. The final relationship of reparametrized Arrhenius equation can be expressed in the form presented in equation (3), which includes the reference temperature. [6]

$$
k=k_{0} \exp \left(-\frac{E}{R T T_{\text {ref }}}\left(T-T_{\text {ref }}\right)\right)
$$

The models designed in this work are based on the knowledge of chemical kinetics. All of them describes the composition of the reaction mixture over time during the transesterification reaction and are based on law of mass conservation.

\subsection{Methanolysis reference model}

The model of methanolysis proposed in [3], which is represented by equations (4-14), was used as a reference model to other suggested models. However, this model was clarified for reactions with other initial conditions, reaction temperatures and dosage procedure. The transesterification described in said publication was catalyzed by TMAH (Tetramethylammonium hydroxide).

The model included further simplifications:

- all reactions are irreversible;

- monoglycerides are neglected due to methanolysis selectivity in case TMAH is used as the catalyst;

- $\quad$ saponification of fatty acid esters is neglected;

- only a single reaction rate constant is describing the saponification of glycerides.

$$
\begin{gathered}
r_{1}=-k_{1, \infty} \exp \left(-\frac{E_{A 1}}{R T}\right) c_{C A T}^{n} c_{T G}^{2} c_{M e O H} \\
r_{2}=-k_{2, \infty} \exp \left(-\frac{E_{A 2}}{R T}\right) c_{C A T}^{n} c_{D G}^{2} c_{M e O H} \\
r_{3}=-k_{3, \infty} \exp \left(-\frac{E_{A 3}}{R T}\right) c_{C A T}^{m} c_{T G}^{2} \\
r_{4}=-k_{3, \infty} \exp \left(-\frac{E_{A 3}}{R T}\right) c_{C A T}^{m} c_{D G}^{2} \\
\frac{d c_{T G}}{d \tau}=r_{1}+r_{3} \\
\frac{d c_{D G}}{d \tau}=-r_{1}+r_{2}-r_{3}+r_{4} \\
\frac{d c_{M e O H}}{d \tau}=r_{1}+2 r_{2} \\
\frac{d c_{F A M E}}{d \tau}=-r_{1}-2 r_{2}
\end{gathered}
$$




$$
\begin{gathered}
\frac{d c_{G}}{d \tau}=-r_{2}-r_{4} \\
\frac{d c_{S O A P}}{d \tau}=-r_{3}-2 r_{4} \\
\frac{d c_{C A T}}{d \tau}=r_{3}+2 r_{4}
\end{gathered}
$$

\subsection{Final suggested model of methanolysis}

It has been examined that the presence of monoglycerides in the reaction mixture is important from the technological point of view and especially in the case of catalyst used in this study the presence of monoglycerides cannot be neglected.

We have tried to involve reversibility to the model, but this feature did not help to describe the reactions studied more accurately and made the mathematical model even more complicated as the number of model constants doubled. For this reason, we considered the reactions to be irreversible as in the reference models.

Since the rate of glycerides saponification may vary, saponification constants for individual glycerides were added.

An important innovation compared to the reference model is the inclusion of reparametrization of the Arrhenius equation into the model. A temperature of 50 ${ }^{\circ} \mathrm{C}$ was selected as the reference temperature for the reparametrization, as the reactions studied were carried out at $40^{\circ} \mathrm{C}$ and $60{ }^{\circ} \mathrm{C}$.

$$
\begin{aligned}
& r_{1}=-k_{1, \infty} \exp \left(-\frac{E_{A 1}}{R T T_{r e f}}\left(T-T_{\text {ref }}\right)\right) c_{C A T}^{n} c_{T G}^{2} c_{M e O H} \\
& r_{2}=-k_{2, \infty} \exp \left(-\frac{E_{A 2}}{R T T_{r e f}}\left(T-T_{r e f}\right)\right) c_{C A T}^{n} c_{D G}^{2} c_{M e O H} \\
& r_{3}=-k_{3, \infty} \exp \left(-\frac{E_{A 3}}{R T T_{r e f}}\left(T-T_{r e f}\right)\right) c_{C A T}^{n} c_{M G}^{2} c_{M e O H} \\
& r_{4}=-k_{4, \infty} \exp \left(-\frac{E_{A 4}}{R T T_{r e f}}\left(T-T_{r e f}\right)\right) c_{C A T}^{m} c_{T G}^{2} \\
& r_{5}=-k_{5, \infty} \exp \left(-\frac{E_{A 5}}{R T T_{\text {ref }}}\left(T-T_{\text {ref }}\right)\right) c_{C A T}^{m} c_{D G}^{2} \\
& r_{6}=-k_{6, \infty} \exp \left(-\frac{E_{A 6}}{R T T_{r e f}}\left(T-T_{\text {ref }}\right)\right) c_{C A T}^{m} c_{M G}^{2} \\
& \frac{d c_{T G}}{d \tau}=r_{1}+r_{4} \\
& \frac{d c_{D G}}{d \tau}=-r_{1}+r_{2}-r_{4}+r_{5} \\
& \frac{d c_{M G}}{d \tau}=-r_{2}+r_{3}-r_{5}+r_{6} \\
& \frac{d c_{M e O H}}{d \tau}=r_{1}+r_{2}+r_{3} \\
& \frac{d c_{F A M E}}{d \tau}=-r_{1}-r_{2}-r_{3}
\end{aligned}
$$

$$
\begin{gathered}
\frac{d c_{G}}{d \tau}=-r_{3}-r_{6} \\
\frac{d c_{S O A P}}{d \tau}=-r_{4}-r_{5}-r_{6} \\
\frac{d c_{C A T}}{d \tau}=r_{4}+r_{5}+r_{6}
\end{gathered}
$$

\section{Experimental measurements}

The models designed in the previous chapter were verified by experiments. The experiments were carried out under different conditions, with special regard to reaction temperature and the method of feedstock dosing.

The samples were taken for analysis during the experiments. Gas chromatography was used to quantify glycerides and esters. Detailed description of the analytical method is available in [7]. The samples were also titrated to determine the extent of saponification as described in [3].

Chemicals used in this study were of analytical reagent grade and were used without any further purification. Commercial rapeseed oil purchased from a local shop was used as the feedstock.

\subsection{Typical procedure}

An amount of $250 \mathrm{~g}$ of rapeseed oil was mixed with methanol (in a 1:6 molar ratio) in a four-way sulphonation flask. The mixture was slowly heated under reflux in a water bath (Thermo HAAKE P5) with constant stirring with a Heidolph RZR 2020 metal stirrer at a speed of $300 \mathrm{rpm}$. After reaching a steady temperature, a catalyst solution $(21 \% \mathrm{KOH}$ in $\mathrm{MeOH}$ in an amount corresponding to the design of the experiment) was injected into the reaction mixture, followed by an increased stirring speed of $2000 \mathrm{rpm}$. This stirring speed ensures that measurement proceeds in the so-called kinetic regime of measurement - details were provided in [3].

Summary of the first experiment: Transesterification of rapeseed oil at $60{ }^{\circ} \mathrm{C}$ using $0.3 \%$ (based on feedstock weight) $\mathrm{KOH}$ catalyst, rapeseed oil:methanol 1:6.

Samples of the reaction mixture were taken at selected time intervals for gas chromatography and titration analysis. The course of the first experiment is depicted in Figure 4. 


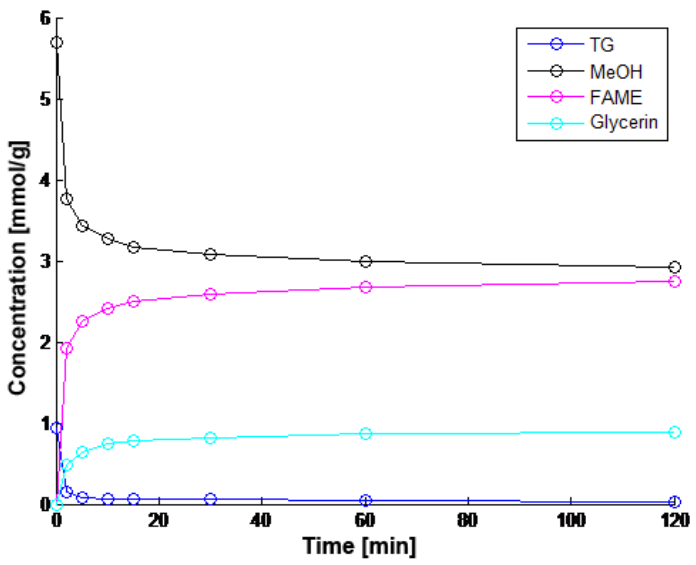

Fig. 4. Course of typical procedure - Concentration of TG, $\mathrm{MeOH}$, Glycerin and FAME.

\subsection{Non-Typical procedures}

\subsubsection{Gradual dosing of catalyst}

This experiment was a test of the gradual dosing of the catalyst to see the reaction behaviour under such conditions. The beginning is identical with the typical procedure; an amount of $250 \mathrm{~g}$ of rapeseed oil was mixed with methanol in a four-way sulphonation flask; the mixture was slowly heated under reflux in a water bath with constant stirring with a metal stirrer at a speed of $300 \mathrm{rpm}$. After reaching a steady temperature of 60 ${ }^{\circ} \mathrm{C}$, the first portion of the catalyst (14.78 g of $2.6 \%$ $\mathrm{KOH}$ in $\mathrm{MeOH}$ ) was injected into the reaction mixture, followed by increasing the stirring speed to $2000 \mathrm{rpm}$ and the second portion was added at a time of 22 minutes (14.73 g 2.6\% $\mathrm{KOH}$ in $\mathrm{MeOH})$.

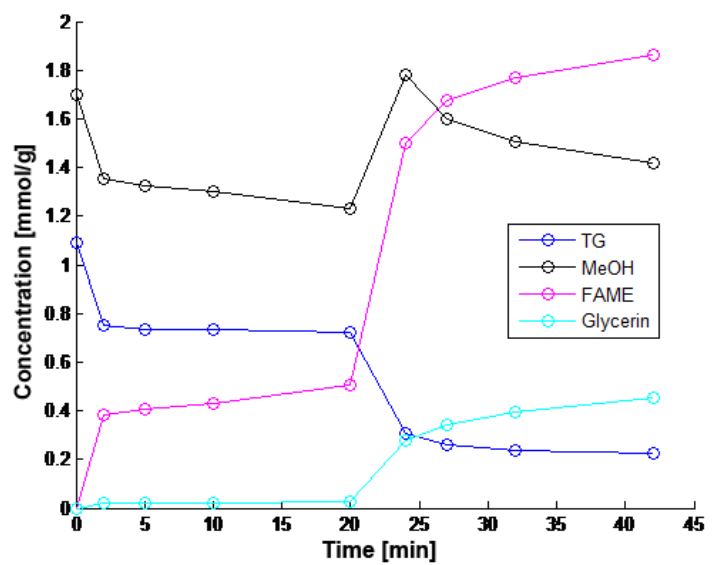

Fig. 5. Gradual dosing of catalyst procedure - Concentration of $\mathrm{TG}, \mathrm{MeOH}$, Glycerin and FAME.

The progress of the reaction evaluated by the analysis of the samples is shown in Figure 5. The second batch of catalyst greatly influenced the course of the reaction. For this reason, the experiment was divided into two distinct parts which were treated separately in finding optimal parameters of the models, as it would be very difficult for the model to record such a change (later addition of catalyst) in the reactions.

\subsubsection{Gradual oil dosing}

The procedure is comparable to the course of the experiment where the catalyst was sequentially dosed, but in this case the catalyst was dosed at the start of the reaction at full dose. The difference will be in the dosage of rapeseed oil, which was divided into two same doses.

The weighed catalyst $(20.5 \% \mathrm{KOH}$ solution in $\mathrm{MeOH}$ corresponding to the design of the experiment) was mixed with weighed methanol (1: 6 , calculated relative to the total amount of oil) in a four-tube sulfonation flask. The mixture was slowly heated under reflux in a water bath and continuously stirred with a metal stirrer at $300 \mathrm{rpm}$. After reaching a steady temperature of $60^{\circ} \mathrm{C}$, a first portion of rapeseed oil $(69.49 \mathrm{~g})$ was added to the reaction mixture, followed by an increase in stirring speed of $2000 \mathrm{rpm}$ and a second portion of oil $(69.60 \mathrm{~g})$ was added at a time of 22 minutes.

As can be seen in Figure 6, at the beginning, the first oil dose practically completely reacted, as seen in triglyceride concentrations, due to the initial high catalyst and methanol. After the second batch of oil, the reaction mixture already behaved as expected, similarly to other experiments, since the concentration of catalyst, methanol and oil was similar.

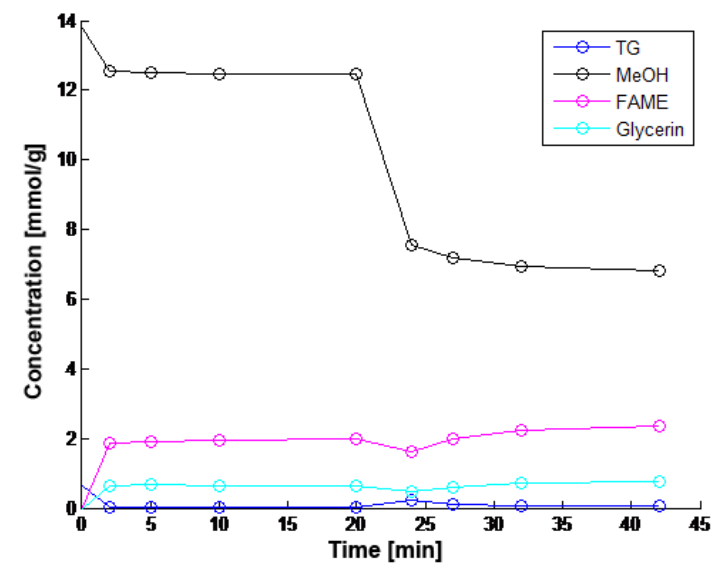

Fig. 6. Gradual oil dosing procedure - Concentration of TG, $\mathrm{MeOH}$, Glycerin and FAME.

\section{Results and discussion}

The reliability of the experimental measurement results was investigated by calculating the sum of glycerides and glycerol (i.e. the total glycerol) from the initial composition of the reaction mixture (i.e. determined by the weights and purities of used chemicals) and the average total glycerol determined by the whole experimental setup (sampling, reaction termination, reaction mixture chromatographic analysis). The results of these calculations were compared to assess the measurement accuracy and minimalize the systematic 
error. The average relative error in total glycerol was 1.4 $\%$ from all used experiments. The comparison of the initial state of the experiment with the analyses of the whole experiment was also applied to the measurement precision confirmation of the total base concentration; the average relative error in the total base was $1.6 \%$ from all experiments.

After considering and testing several models, the experiments with the atypical procedure were split at the time of the second dose (22nd minute). It resulted in two distinct data sets with atypical initial conditions. It should be emphasized that the used mathematical models assume a homogeneous reaction system and the reference model has been validated on experimental data obtained at a constant ratio of methanol to glycerides. The experimental conditions used in this work varied, as well as the mentioned ratio of methanol to glycerides, to a large extent.

Optimal values of all model parameters were determined using the simplex method implemented in MATLAB software. The sum of squares of relative weighted deviations (29) was chosen as the objective function.

$$
f_{U}=\sum_{i=1}^{i=n} w_{i}\left(\frac{c_{i, C A L}-c_{i, E X P}}{c_{i, E X P}}\right)^{2}
$$

Table 1. Suggested optimal constants of the model.

\begin{tabular}{|c|c|c|c|}
\hline $\mathrm{i}$ & $k_{i, \infty}[1 / \mathrm{s}]$ & $\mathrm{i}$ & $E_{A i}[\mathrm{~kJ} / \mathrm{mol}]$ \\
\hline 1 & 6.20 & 1 & 41694.46 \\
\hline 2 & 19.53 & 2 & 67892.17 \\
\hline 3 & 28.06 & 3 & 52200.42 \\
\hline 4 & 0.49 & 4 & 63107.97 \\
\hline 5 & 0.64 & 5 & 43195.56 \\
\hline 6 & 0.64 & 6 & 13455.32 \\
\hline- & - & - & - \\
\hline $\mathrm{n}[1]$ & 0.89 & $\mathrm{~m}[1]$ & 0.84 \\
\hline
\end{tabular}

As can be seen in Table 1, optimal model constants have been found by the described method. Since this model describes a system that involves not only the direct reactions but also multiple side reactions, optimization of the model constants values means to be looking for a minimum in a 14-dimensional space, which is not a simple task. In the view of this figure, the demand for reasonable model simplification described in chapter 2 is obvious.
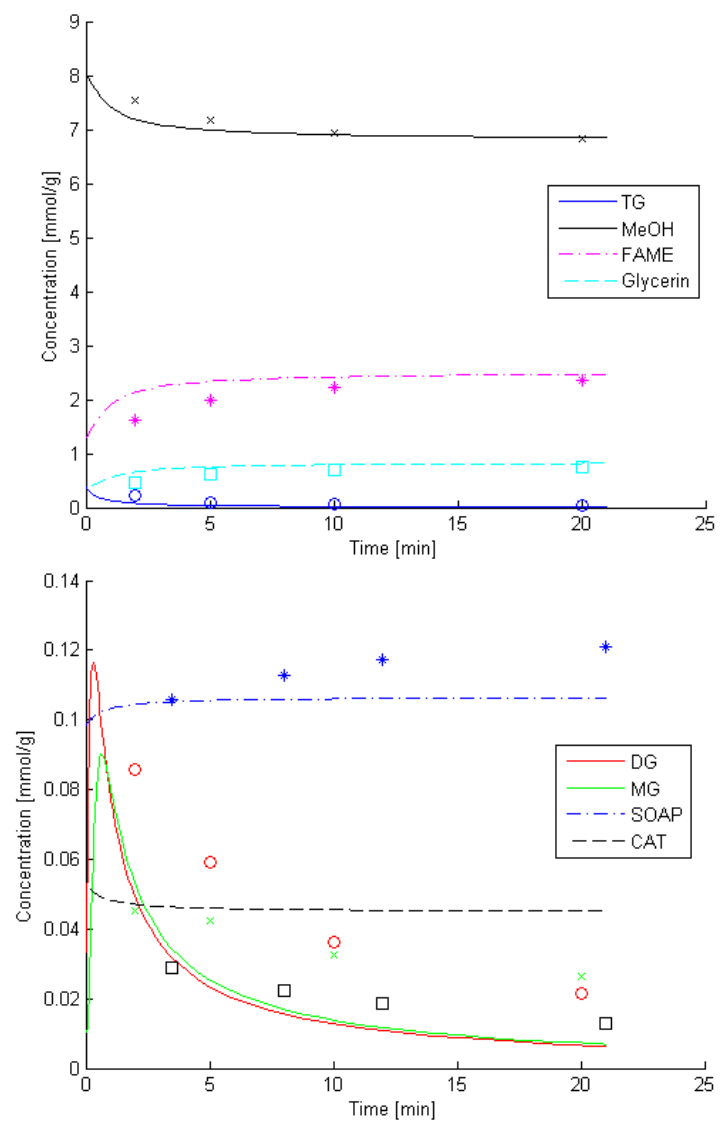

Fig. 7. Comparison of experimental data from gradual oil dosing procedure (points) and final model prediction (lines).

Figure 7. shows experimental data from the second part of the gradual oil dosing procedure with the final model prediction. For comparison, measurements from the typical procedure with the model assumption are depicted in Figure 8. As can be seen, the model predicts the reaction mixture composition very well during the reaction, considering the width of the reaction conditions to which the model was optimized. Average model relative deviation from measured experimental data was calculated at $25.2 \%$. The deviation may seem insufficient, but it is a significant improvement compared to the reference model. The average deviation for the reference model was $42.3 \%$.

However, the model-optimizing algorithm has minimized the deviations for certain substances in the reaction mixture. A higher priority in optimization was given to the fatty acid methyl esters and an extent of saponification. These components are the most important in biodiesel production in the view of the final product purity. 

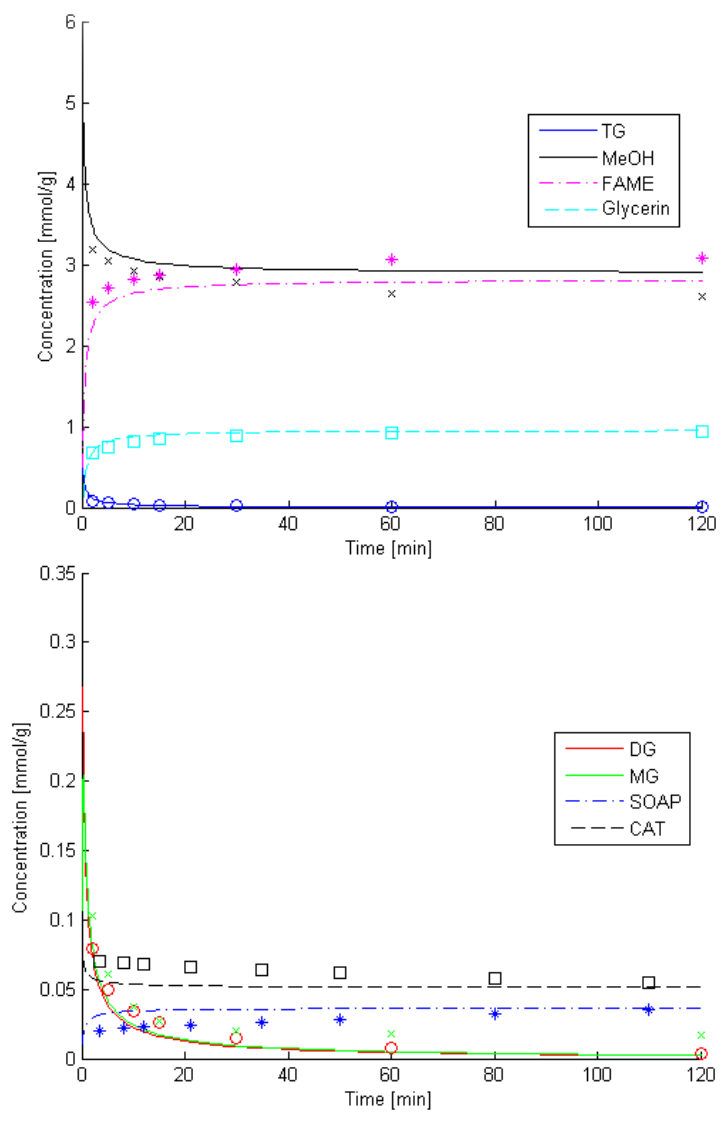

Fig. 8. Comparison of experimental data from typical procedure (points) and final model prediction (lines).

\section{Conclusion}

The purpose of this work was to propose a model of transesterification of rapeseed oil catalyzed by $\mathrm{KOH}$. The model also considers the side reaction, which is related to the utilization of a basic catalyst. The final model was adapted according to the theories of chemical reactions and available literature, and employs reparametrization of the Arrhenius equation with a selected reference temperature of $50^{\circ} \mathrm{C}$.

Several experimental reactions were carried out in order to refine and verify the suggested model based on the reference model of methanolysis. The experiments were conducted under different reaction conditions, with special focus on the reaction temperature and the initial amount of oil, methanol and catalyst. The range of reaction conditions was extensive, as various feedstock dosing methods were used. Therefore, the average model relative deviation from experimental measurements 25.2 $\%$ presents very good result, especially when we take the complex nature of studied system into account.

To conclude, the final model can be useful to simulate the reaction under various reaction conditions with the focus on prediction the concentration of fatty acid methyl ester and an extent of saponification in the time.
This work was supported by the Ministry of Education, Youth and Sports of the Czech Republic within the National Sustainability Programme project No. LO1303 (MSMT$7778 / 2014)$ and also by the internal project No. RVO/CEBIA/2019/003..

\section{References}

1. M. Canakci, J. Van Gerpen, ASAE Annual Meeting Papers. ASAE Paper No. 01-6049, 29 (2001)

2. J. Van Gerpen, Fuel Process. Technol., 86, 10 (2005)

3. J. Pecha, L. Sanek, K. Kolomaznik, Chem. Eng. Sci., 288, 680-688 (2016)

4. S.N. Csernica, J.T. Hsu, Ind. Eng. Chem. Res., 51, 6340-6349 (2012)

5. J. Novak, Fyzikální chemie: bakalářský a magisterský kurz, (2008) (czech book)

6. M. Schwaab, J.C. Pinto, Chem. Eng. Sci., 288, 680688 (2007)

7. L. Sanek, J. Pecha, K. Kolomaznik, J. Sep. Sci., 36, 1029-1036 (2013) 